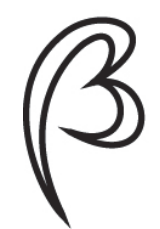

Bureau d'économie

théorique et appliquée

(BETA)

UMR 7522

\title{
«Are the risks of being creative manageable? The case of public research in Hard Science »
}

\author{
$\underline{\text { Auteurs }}$ \\ Ylenia Curci, Mireille Matt, Isabelle Billard, Thierry Burger-Helmchen
}

Document de Travail $n^{\circ} 2017-30$

Bureau d'Économie

Théorique et Appliquée

BETA - UMR 7522 du CNRS

\section{BETA Université de Strasbourg}

Faculté des sciences économiques et de gestion

61 avenue de la Forêt Noire 67085 Strasbourg Cedex

Tél. : +33 (0)3 68852069

Fax : +33 (0)3 68852070

Secrétariat : Géraldine Del Fabbro g.delfabbro@unistra.fr

BETA Université de Lorraine

Faculté de droit, sciences

économiques

et de gestion

13 place Carnot C.O. 70026

54035 Nancy Cedex

Tél. : +33(0)3 72742070

Fax : +33 (0)3 72742071

Secrétariat : Sylviane Untereiner sylviane.untereiner@univ-lorraine.fr 


\title{
Are the risks of being creative manageable?
}

\section{The case of public research in Hard Science.}

\author{
Ylenia Curci ${ }^{1}$, Mireille Matt ${ }^{2}$, Isabelle Billard ${ }^{3}$, Thierry Burger-Helmchen ${ }^{1}$
}

'BETA, CNRS-UMR 7522, Univ. Strasbourg, 67000 Strasbourg, France, ${ }^{2}$ GAEL, INRA, CNRS, Grenoble INP, Univ. Grenoble Alpes, 38000 Grenoble, France,

${ }^{3}$ CNRS, LEPMI, Univ. Grenoble Alpes, 38000 Grenoble, France

\begin{abstract}
This study analyses the risk associated to creative research in the field of Hard Science. The theoretical framework in which the research question has been developed affirms that researchers' creativity and their risk aversion are negatively correlated. In the analysis of two case study, we found that many of the sources of the different risks perceived by researchers are generated by a misfit between scientists' goals and mission of the organization in which they operate. In this research, we found that scientists show aversion for a great variety of risks, which mainly change according to the research field, to the nature of the research (basic vs applied), to the composition of the research team and to the type of research activities (experimental vs theoretical). This paper develops a categorization of the observed risks and proposes the exploitation of some resources in order to decrease the impacts of the risks on researchers' creativity.
\end{abstract}

Keywords: creativity management, leadership, economics of science

JEL Codes: D23, L30, M19, O30 


\section{Introduction}

This study aims to investigate the setting in which creative research thinking occurs, at the individual and team level, in Hard Science ${ }^{1}$.

During the last century, creativity has been studied from different perspectives by scientists working in several fields. Many of them have provided a slightly different definition of creativity along the years ${ }^{2}$, although the majority agrees on defining creativity as the ability to do new things which have some utility (Amabile, 1996). In the field of science, creativity has been contextualized by Hollingsworth (2004) and Simonton (1988, 2004), and defined as the "knowledge and capabilities that are new, original, surprising and useful".

In a context of indefinite success' potential of ideas and risk aversion of agents, Sternberg and Lubart (1991) elaborated a theory in which being creative is comparable to being hazardous investors. According to their "Investment approach to creativity" (Sternberg et al., 1997), creative people are good at buying low (unpopular ideas) and selling high (persuading the crowd of their validity). In this approach, creative people possess specific attributes: in particular, they are risks takers which are able to exploit different resources to reduce the negative outcomes of risks. In this matter, the theory lists six resources (intellectual abilities, knowledge, styles of thinking, personality, motivation, and environment), but it does not provide a specific definition of risk, neither clarify in what way risks may influence creativity.

In the economic and social psychology literature, many authors agree that the level of risks aversion can influence creativity of individuals and teams, and provided a precise description of the circumstances in which people take risks and why, especially from an employees' point of view (Dewett, 2004). To our knowledge, the story of which are the risks affecting specifically the

\footnotetext{
${ }^{1}$ Which is defined as "any of the natural or physical sciences, as chemistry, biology, physics, or astronomy, in which aspects of the universe are investigated by means of hypotheses and experiments".

${ }^{2}$ For an extensive review of creativity definitions see Ochse, 1990
} 
research in the field of Hard Science, as well as the effects they may have on researchers' creativity, is so far untold.

In this paper we endeavor to address this question by the analyses of two case study in which we found that researchers working in public institutions, operating in absence of profit motives, use to organize their working according to goals primarily related to intrinsic motivations. This evidence is found unaltered despite the fact that in recent years, some institutional and organizational change of the public research setting has introduced financial and performance constraints which have modified the approach that scientists have towards creative research projects. By behaving in a creative way, researchers perceive a variety of risk mainly generated by a misfit between their objectives and the institutionalized mission of the modern public research sector.

This work focuses on interviews with researchers specialized in three areas of interest in the broad Chemistry domain (Electrochemistry, Physical Chemistry of Materials and Physical Chemistry of Interfaces) and elaborates on them with the final aim to disentangle the role that risks play in affecting researchers' creativity. The final aim of this work is to help the individuation of the settings that facilitate/endanger risks taking behaviors, both at individual and team level.

The remainder of this paper is organized as follows: section 2 shortly reviews literature on scientific creativity and risk management; section 3 illustrates the methodology adopted for case study, data selection and data analysis, section 4 presents the outcomes of the qualitative analysis and proposes a categorization of the observed risks, the last section discusses some of the evidence arising from the case study and presents some policy recommendations.

\section{Background and literature review}


The father of the modern notion of creativity is probably Henri Poincare who, in the 1908 noted: «to create consists of making new combinations of associative elements which are useful [...] the most fertile will often be those formed of elements drawn from domains which are far apart» (cf., Mednick, 1962).

The study of creativity has been growing considerably in the second half of the XX century. Since then, many social scientists have focussed on creativity antecedents ${ }^{3}$, creative $^{2}$ thinking, outcomes of creative works, creative processes (Caniëls et al., 2014) and creative individuals, but, despite the recognition of its economic importance, very little work has been done in the economic literature (Charness and Grieco, 2013). In fact, from Schumpeter on (1939), researchers have chiefly elaborated on how firms and other entities manage their innovation processes, and how innovation contribute to growth and development of nations: in economics, the history of generation of new ideas, what foster their formation and growth, as well as the different routes ideas have to go through to dethrone the status quo, remain largely neglected. Nevertheless, in the recent years, the role of creativity has gain importance, in particular in sectors where the output of the production process is represented by the creation of knowledge (Caniëls et al., 2014).

During the second half of the XX century, scientist developed numerous methods to capture and quantify creativity. Building on Guilford's seminal work (1950), Ellis Paul Torrance (1988) tried to measure creativity by comparing convergent and divergent thinking. From the evaluation steps of his TTCT (Torrance Test on Creative Thinking), it is possible to extrapolate some of the features that, according to many scholars in this and the last century, characterize creative thoughts: novelty, uncommonness (hence, divergence from the crowd), and usefulness. ${ }^{4}$

\footnotetext{
${ }^{3}$ Both at individual and team level. See for instance Shalley et al., 2004 and Barzack, 2010.

${ }^{4}$ In fact, the TTCT scores creative responses to external stimulus according to i) meaning and relevance, ii) statistical rarity, iii) frequency of spontaneous shifts from one category of meaning to another, and iv) detail and specificity of the response.
} 
Starting from the works of Torrance, which focused on creative responses, creativity has been studied from various angles. A stream of literature questioned whether there might be a category of gifted people characterized by divergent thinking, thus focussing on creative individuals. Along with the TTCT, personality tests have been formalized to detect relevant personal features of creative persons, such as «independence of judgment, self-confidence, openness to experience, balanced personalities, attraction to complexity, aesthetic orientation, and risk-taking.» ( Charness and Grieco, 2013, pp.3).

One of the most popular shifts from creative objects and responses towards creative individuals is represented by the work of Sternberg and Lubart which, in 1991, developed an "investment approach to creativity". The theory delineates how gifted people express their creativity by diverting from the humdrum thinking of the crowd and defying its interests. According to this theory, creative thinkers are good at selling ideas in the same way that investors are good at buying at low prices and selling at high prices: creative people make up ideas that are similar to stocks that the public undervalue or reject. Even though creative ideas are both novel and valuable, they are often considered weird, of no use, and even crazy. At the same time, creative people are frequently distrusted and perhaps even disdained and mocked. The skill of a creative person lies with the ability to identify growth potential in unknown or unwelcome ideas and persuade the crowd that they represent a valid and superior way of thinking. More importantly, buying low implies a certain degree of risk taking by individuals.

In the economic literature, risk has been defined by Frank H. Knight almost 100 years ago as a circumstance where one can assign a probability to different possible outcomes of a decision he has to make (Knight, 2012). In project management literature, risk is defined as an «an uncertain event or condition that, if it occurs, has a positive (opportunity) or negative (threat) impact on project objectives. » In this work, we consider creativity-related risks every circumstance the researchers have spontaneously acknowledged as a threat to their professional creativity. 
Beyond Sternberg and Lubart, many other authors agree on the key role that risk aversion plays in determining creativity. Staw (1995) argues that the path of generation of new ideas involves a lively process in which ideas are often discharged because perceived as bizarre, inappropriate, impracticable or too risky. Risky and creative behaviors have been linked by several scholars (Carver \& White, 1994; Keltner et al., 2003; Lee et al., 2004; Mainemelis, 2010). Elaborating on the same line, many claims that the higher the risks the more radical the creativity (Benner \& Tushman, 1993; Smith \& Tushman, 2005) and that sharing risks with a group of people enhances creativity (Glover, 1997).

Building a parallelism between the Investment theory of creativity and the case of research in Hard Science in the current institutional and organizational setting, to be considered creative, scientists should convince the crowd of the validity, originality, novelty, and utility of their work (Hollingsworth, 2004; Simonton, 2004). Nowadays the scientific crowd is dual. The first one is represented by "evaluators" (journal referees, editors, conference peers, patent examiners, funding entities, etc.), while the second is represented by peers and other agents that can make use of the research (other scientists that can draw on the new state of the art, firms licensing patents, etc.). The first crowd usually judges research projects and outcomes by using criteria such as plausibility and validity on the one hand; and originality and novelty on the other hand. For this reason, creativity in science suffers from a well-acknowledged tension between the first two criteria, which call for conformity, and the last one, that encourages dissent (Heinze, 2009).

In this paper, we claim that, when favoring originality and novelty at the expense of plausibility and validity, thus defying the conformity of the crowd, scientists take the risk of "buying low".

In Sternberg and Lubart's framework, when creative people take the risks by buying low, the negative outcome of this attitude can be mitigated by six resources: intellectual abilities, knowledge, styles of thinking, personality, motivation, and environment. In the six resources 
individuated by the two authors, environment is among the much debated, even contested, and it is still calling for an investigation. Environment refers to everything which is external to the individual, and that can be supportive, neutral or detrimental for creativity (Stenberg, 2006). In the social psychology and organizational literature, where great attention has been given to group creativity instead of creative individuals, creative teams and methodical creative processes have been analyzed in order to individuate the features of environmental resources that can facilitate creative outcomes (Woodman, 1993). Heinze (2007a, 2007b, 2009) studied creative research in one of the most dynamic field of Hard Science - nanotechnologies - both at individual and team level, and then focussed on environmental factors that have characterized them: he took a "topdown" approach to identifying the determinants of research creativity by surveying some "research stars" who were deemed to be the research leaders of creative scientific accomplishment in the nanotechnology domain. The aim of his work was to investigate the institutional and organizational setting in which the identified creative scientific accomplishment took place. We build on Heinze's work (Heinze et al., 2009) to investigate the risks perceived by researchers in the performance of creative projects with two aims: first we provide a classification of the risks emerging from researchers' experience and second, we observe whether they (consciously or unconsciously) exploit any resource to mitigate the detrimental effect of such risks. Conversely to Heinze approach, in this work, we focus on the ability of researchers to think creatively with the only constraint that such creative thinking resulted in a peer-reviewed scientific publication. Therefore, we took a rather different approach from Heinze and his colleagues, which focused on the creative outcome of the research and investigated what were the determinants of that outcome. On the contrary, in our work, individual researchers were asked to indicate what triggers creativity from their perspective based on their recent experiences in terms of self-evaluated creative research. This is to ensure that we do not only identify the setting in which a creative research product has been developed, but also that we investigate the setting in which the researchers think in a creative way, and if in doing so they perceived any risks. 
Thus, in this work:

1. we provide a definition of creativity which will be used to individuate creative scientists,

2. we adopt a definition of creativity-related risk,

3. we look for the variety of creativity related risks experienced by creative researchers,

4. we build a categorization of the risks observed, and

5. we investigate whether they exploit any resource to mitigate such risks.

\section{Methodology}

The investigation has been carried out by means of two case study ${ }^{5}$. A two-step analysis has been performed on two French Laboratories, two public research centre that brings together expertise in both basic and applied research in most areas of electrochemistry, particularly in the production and storage of electrochemical energy, involving process engineering. The two labs count 80 permanent staff and hundreds of postdocs, Ph.D. students and interns. The laboratories are partners in several competitiveness clusters, have exchange programmes with foreign universities and undertake projects jointly with the industrial sector.

The first step in the investigation consisted of a web survey which was carried out in 2015. Out of 470 invitations (permanent staff excluding administrative personnel, plus nonpermanent researchers, including Ph.D. candidates), we received 161 reliable and complete responses (with a response rate of $34.2 \%$ ). The sample is composed for $70 \%$ by males and includes 38 foreigners scientists. The survey consisted of 104 questions divided into 9 sections, each one focusing on Personal Information, Background and area of interest, Role within the

\footnotetext{
${ }^{5}$ An extension of the data collection will be carried out at the end of September in another French research facility.
} 
Institution, Relationship with the industry, Leadership, Publications and co-authors, Patents, Collaboration and collaborators, and Failures in collaboration.

Step two consisted of 27 face-to-face interviews conducted in June and September 2015. The people for the interview were selected using two parameters: 1) people nominated as creative people in the survey conducted in step one by at least two participants, and 2) everyone but Professors and Research team leaders with high numbers of publications. The reason for the first parameter relies on the following argument: a creative person is someone who has been «recognized by expert opinion as having contributed something of original value to the culture» Ochse (1990, p. 4). Hence appropriate observers (people familiar with the chemistry domain) should independently agree on judging a person as creative (Amabile, 2012). The reason for the second parameter seeks to correct the intrinsic bias of the first one. Acknowledgment of creativity in a domain in which knowledge and reputation are extremely important frequently leads to identifying only very experienced people as being creative. This may result in the biased conclusion that young people are not creative. Hence, we included highly productive scientists (with more than 10 publication per year) in the sample, ending up including several postdocs in the interview.

In the nomination part of the survey we did not ask directly to provide a name of a creative person. Conversely, we ask to indicate a colleague that the respondent considers as a guidance, an inspiring researcher, and then to illustrate the reason for such choice by selecting at most 2 of the given options. The possible answers to the multiple-choice questions have been classified according to different categories (authority, age, fundraising, charisma, and creativity). Among 161 people, $45 \%$ of the respondents indicated a person they consider as being creative by selecting at least one of the following:

- Because he/she has always new ideas

- Because he/she elaborates valuable scientific questions out of weird experimental results 
The interview was divided into two parts. The first part involved asking researchers to self-assess their own work in terms of creativity and compare their most creative works with their most acknowledged ones (in term of citations). In particular, we asked the following question: "Could you please tell me the title of the article related to your most creative research?" Once we received the titles, we immediately checked whether the article received in Web of Science the highest number of citations among the publications of the interviewed researcher. The next question was "Why you think this is the most creative? What characterizes it the most?" When the article they nominated as the most creative was not the one which received more citations, we asked the following question "Why you think this other article is the most cited? What characterizes it the most?"

Part two included questions related to their most creative research work (based on selfassessment).

In particular, we asked them to describe each stage of the research from the creation of the research question to the end of the project (although some of them were still ongoing) ${ }^{7}$. The protocol included a specific question about risks, but it was asked at the very end of the interview when researchers did not spontaneously talk about the issue. The interviews have been recorded and transcribed. We present in the next section the risks and resources identified from the analysis of the texts.

\footnotetext{
${ }^{6} \mathrm{~A}$ published paper provide some sort of insurance that the research was found novel and useful by at least few peers and it contributed to the culture (Ochse, 1990)

7 The main variables of the second part were: the different steps you go through when you decide to do a project and how to do it, size of the research group, composition of the research group in terms of positions of the people participating, composition of the research group in terms of nationality of the people participating, type of leadership, quality of leadership, scope of the research topic, duration of research, need and capacity of the project to access external/expansive resource, need of the project to access a large variety of technical skills, structure of the organization hosting the research, its size, its reputation and visibility, its capacity to access a large variety of technical skills, autonomy in choosing the research topic, clarity of research goals, independence of decision-making of the research team, ability to extend deadlines, ability to hire new people, flexibility to unexpected changes, perceived risk in undertaking the research and main constraints.
} 


\section{Results and discussion}

In this study, we focus on researchers specialized in different areas of interest in the broad Hard Science domain. These areas allowed us to observe some distinguishing characteristics of research specializations in Hard Science for which the theory of creativity as an investment seems to be suitable. In fact, the idea of "buying low" always embeds a certain degree of risk, although is not clear what are the effects of risks on the different components of scientific creativity. The outcome of the interviews provided a variety of feedbacks that we will endeavor to classify accordingly to the existing literature.

In our sample, public researchers described their work as being inherently risky: «even when one searches by not taking any risk, there is always the risk, that he finds nothing».

Although aware of the risks, many researchers declared they were not seeking to minimize them and they provided many explanations for this. Nevertheless, two main points of evidence arose from the first part of the interview as almost all researchers acknowledged the following: i) Ph.D. students need to publish before defending their thesis and subsequently; ii) mentors and tutors tend to minimize the riskiness of projects (or a part of it) when Ph.D. students are involved. In this sense, the application of project management methods to research (that is reported as a great source of risk later in this section) allows experienced scientists to pursue creative and risky research by confining "fragile" researchers to more secure tasks. In fact, by applying project management methods to scientific research, projects have to be broken down into pieces, each one entailing a different degree of risk.

With only one exception, all the interviewed people reported a variety of different risks and were able to discriminate between "impossible to avoid" risks and those that can be avoided in some way. Some researchers autonomously talked about risk by replying to the second question. With rare exceptions, all researchers were able to identify a specific category of risk that 
characterized their creative research. In the following section, we present all the risks reported and, where individuated, the resources applied by scientists to minimize them.

\subsection{Risks and resources identification}

\section{Experimental risks}

Source: a controversial risk is one related to the so-called "physical risk". Some research teams work with potentially harmful (e.g. radioactive) material and they need to safely solve the paradox of being creative in domains in which in general they must not be. When research implies a certain degree of danger, creativity may put researcher at risk. Generally speaking, creativity is not welcome when you mix radioactive substances for nuclear waste reprocessing studies. During the interview, the only person reporting physical risks has been the one in charge of lab safety. None of his colleagues talked about it, but in the interviews, we did not manage to disentangle whether this is because they all tend to minimize risk by default or because they take for granted that they continuously have to face it.

Another experimental risk refers to the lack of knowledge about new instrumentation. In our sample, some experienced researchers affirmed that you can never put a Ph.D. student working on some new platform, because you cannot predict whether it will work or not, neither you can conjecture on its precision and efficiency. As mentor, they said they have to guarantee the full educational path to Ph.D. candidates, which includes experiment, analysis and interpretation. New instrumentation cannot assure this path, so it is too risky to rely on it for a Ph.D. thesis. 
A similar experimental risk is linked to the radicalism of the innovative content of the research and thus to the uncertainty of outcomes. When you are doing something extremely new (e.g. how captors behave in a big surface), nobody knows what to expect because the knowledge base is not sufficient to provide reliable previsions. In that case, knowledge does not exist, and intellectual ambiguity in the norm.

Resource: High levels of knowledge can substantially reduce experimental risks. A full and Omni-comprehensive knowledge of the experimental material can allow foreseeing any possible outcome of the new procedures. In this sense, because knowledge is expanding its base and its complexity, is always more difficult for an individual, and for research teams in which individuals share the same background, to master the whole knowledge needed. From the interviews, we observed that a great resource is represented by the so-called "silent designers" of scientific research, which is support personnel capable of creating models/methods/original experimental protocols. Creative engineers have the ability to provide safer and more effective instrumentation to perform the research and represent a valuable resource to control experimental risks.

\section{Resources requirement risks}

Source: most of the participants in the interview acknowledged that they tend to buy low easily when buying low is not costly in terms of time, financial and physical resources. In the theory of Creativity as Investments, buying low refers to the concept of "unpopular ideas". In Chemistry and Physics as in all Hard Science, buying bizarre/extravagant ideas can be both very costly and very cheap in economic terms. In our sample, we included scientists working in different areas of interests, hence allowing us to disentangle the determinants of creativity between research projects for which the financial resources represent an extremely different issue. For experimental chemists, work tools are usually consumable materials and chemical reagents, as well as extremely expensive instrumentations, whereas, for chemistry modelers, the 
tools of the trade are basically personal computers. Experiments usually require time for phenomena to occur, and students and other personnel who take care of measurements, characterizations, data collection and so on, whereas modelers can work in pretty small groups with limited time needs. The challenge to be creative is enhanced when the research project requires huge financial, time and human resources.

Resources: The researchers interviewed demonstrated a crucial ability in handling this kind of risk by balancing their "projects portfolio" between tasks needing a different amount of resources - thus implying a different degree of risk. Researchers made very clear that they have to "assure a certain quantity of production" but they are also aware that there is not a strict link between the tangible outcome of the research (publications, patents, protocols, etc.) and the money spent on the whole project.

\section{Resources availability risks}

Source: in our sample, many recognized that an extra source of risk has been introduced by ad hoc financial provisions. Modern scientific research is more and more organized in projects for which aims, resources and timing must be precisely specified (Gido and Clemens, 2009). However, scientists - in particular those oriented towards basic research, lament that ad hoc funds procurement do limit their creativity. Such limitations occur in a threefold way.

First, when their primary research topic is not considered "fancy" at the decisionalpolitical level, researchers struggle to get money to perform their own projects. In those cases, the majority of scientist ends up applying for unappealing (to them) research subjects, from which they save resources to pursue their own scientific interests, and face the risk of being caught as well as the risk of undermining the original project.

Secondly, even when personal inclination and fashionable subjects overlap, researchers admit that they are not able to specify reliable development stages and plausible outcomes of 
research projects, and they always risk not to fulfill the requirements of projects calls. As Jensen noted, such ambiguity and inconsistency of aims persist for a physiological period of time, after which projects can reach a clear degree of specificity (Jensen, 2011). When researchers apply for funds, they are trapped between what they would like to experiment, and what they can feasibly deliver. In the "investment" approach to creativity (Lubart \& Sternberg, 1995) periods of intellectual ambiguity are essentials. Creative personality tolerates ambiguity and acknowledges it as «necessary during those periods of creative endeavor in which things are not quite fitting together». Many PI (principal investigators) described the phases of their projects application procedures as a funnel process in which they discharge too risky ideas and unreliable (although creative) collaborators. In 2001, Koen et al. provided a view of the innovation process divided into three steps: the fuzzy front end, the new product and process development, and commercialization". The fuzzy front end phase is characterized by a "go-no go" process in which ideas are developed or rejected according to their apparent potential and degree of risk. In the sample, scientists reported that when funds are not related to the provision of a specific tool or solution to a practical problem they are quite protected in case they realized they cannot comply with what agreed in the grants documents, because scientific uncertainty is somehow contemplated even in private transactions. Moreover, discovering that «something does not work, is considered a discovery indeed». The story changes when the funds are linked to a predetermined outcome: in these cases, risks minimization is the primary objective, even at the expense of creativity.

Thirdly, some researchers declared that they are simply not interested in the application their research may find outside the lab, and they affirm that their more creative works are currently (and may remain in the future) unusable for whatever purpose. Nevertheless, Amabile relates creativity to the development of new ideas or outcomes that are appropriate to some goal (Amabile, 2012). During the interview, when we highlighted that creative work should have some

\footnotetext{
${ }^{8}$ While the last two have been extensively analysed, the former is still confined into the Rosenberg's "black box" (Rosenberg, 1982; 1994).
} 
utility, we systematically listened to the following argument: in the domain of science, the utility seems to refer to a specific (or also vague but at least existing) aim of research ideas. By definition, basic science and applied science have two different objectives: while the latter is unequivocally related to the research of one or more solutions to a given problem, the former is chiefly devoted to the comprehension of phenomena. When comprehension itself is the final aim, is not important whether it may find an application in the industrial sector, neither now or in the future. In our sample, many researchers working exclusively in basic science seem to acknowledge their research as valuable, even when it does not display any a priori utilitarism. To clarify this point, one researcher stressed his opinion by stating that his Science is for Science's Sake, as occur in arts ${ }^{9}$. As far it is able to describe and sometimes explain the reality, as well as it concludes or stimulates the debate over the studied phenomena, their work has an indisputable dignity.

Resources: in the interviews, we found that the most used resources used to cope with financial availability related risks are style of thinking and personality. On the one hand, "cheating" on budgets and timesheets or making scale economies on materials and instrumentation is a widely spread habit of researchers facing funds constraints. They devote some of their time and resources to the agreed low-risk project, while they keep conducting risky or not fancy projects under the counter. On the other hand, charismatic and eminent scientists, when they want to favor a specific topic or raise money for risky projects, make a step into political contexts or exploit their influence on the industrial sector. In this cases, personality plays a vital role in persuading donors and investors to direct funds and to accept a high degree of risks.

\footnotetext{
${ }_{9}^{9}$ Art for art's sake: « The phrase expresses the belief held by many writers and artists, especially those associated with Aestheticism, that art needs no justification, that it need serve no political, didactic, or other end. » (Encyclopaedia Britannica)
} 


\section{Power concentration risks}

Source: a further risk reported during the interviews is represented by the possibility that you are stepping on your referee's toes. In certain fields, researchers suffer from a sort of obstructionism with respect to the dominant mainstream scientific community. As argued by many authors in the literature of creativity, the creative individual should be able to support her own convictions, even when she has to go against the crowd (Amabile, 1983; Barron \& Harrington, 1981). Although it might be easy to promote niche literature once you have robust results, in a field where experts called to judge the merit of a project proposal are part of the scientific crowd that one intends to defy, getting funds to perform the research become almost impossible.

Resource: the obstinacy of scientists to plumb and to exploit pluralities of environmental resources become essential to guarantee the survival of non-mainstream research lines. So far an important role in this sense has been played by top-down annual research funds. However, those resources are becoming scarcer every year.

\section{Motivational risk}

Source: to the second question in the interview, almost all the respondents described their most creative research work as if there were a parental relationship between them. Sentences like "it was my baby", "I was his father", "I took care of it from when it was in the cradle", were continuously repeated during the interviews. Conversely, scientists declare they, and the research outcome, were less creative when there was no emotional involvement in the work.

Resource: the impact of this personal attachment to ideas on their risk-taking inclination can find a possible explanation on what Stenberg and Lubart called motivational resources. Motivational resources refer to the motivation one has to use the other resources for creative purposes. 
According to organizational, sociological and socio-psychological literature, creativity can be influenced by intrinsic motivators or extrinsic incentives. Intrinsic motivators, which have been recognized as the most powerful in affecting creativity, are characterized by the ability to draw individuals' attention on the creative task solely. Intrinsic motivators include curiosity, passion, self-realization, sense of duty and many others (Golann, 1962; Amabile, 1983; 1985; Staw, 1989). Some authors claim that intrinsic and extrinsic motivation have a cumulative effect while many others argue that people tend to be more creative in the absence of extrinsic rewards, such as money and recognition because they subtract attention to the task and re-direct it towards final goals. In such cases, the task becomes just a means to an end.

The discussion on the detrimental effect of extrinsic rewards and on the circumstances in which the latter can "crowd-out" intrinsic motivation, is old but still ongoing (Deci, 1971; Deci et al., 2001, Frey \& Jegen, 2001; Lazaric \& Raybaut, 2014). In an experimental research, Curci et al. (2017) have empirically demonstrated that the introduction of financial incentives to collaborate has a detrimental effect on the creative content of the output of such collaboration. In this debate, economists are quite skeptic about the possibility that money can disincentivize agents. Nevertheless, as clarified by Kreps, the argument in favor of this position is quite powerful: «...if an employee undertakes some effort without the spur of some extrinsic incentive, he will rationalize his efforts as reflecting his enjoyment of the task. And since he enjoys it, he works harder at it. But if extrinsic incentives are put in place he will attribute his effort to those incentives, developing a distaste for the required effort.» (Kreps, 1997: 362). Moreover, he argues that risk aversion pushes workers towards safer tasks and behaviors. According to Frey (1997), the crowd-out effect caused by extrinsic rewards and any other external intervention can make individuals less responsible and self-determining, weaken their self-esteem by not valuing their dedication to the project and competence, and deny the possibility to demonstrate their intrinsic motivation. 
Beyond creativity, Hideo and Nagaoka (2011), found empirical evidence that intrinsic motivators play a crucial role in determining researchers' productivity as well. In their analysis on Japanese high-tech firms, the authors show that two intrinsic motivators - taste for science and taste for challenges -are the major determinants of the inventors' productivity.

\section{Reputational risk}

Source: although French researchers acknowledged that their jobs positions were never at risk $^{10}$, people stress there is an intrinsic reputational risk in not delivering what you have promised in due time. Reputational risks may also limit another attribute of creative personality: perseverance. Some of the interviewed scientists reported that when they struggle to get good results from their research (even when they are extremely confident about it) they tend to sadly shut down the experiment because of the fear to be held up due to resources and time waste.

Resource: the utility-oriented scientific paradigm sometimes shifts researchers' obligation from means to results. This circumstance has made researchers worrying about something they were not used to put into the equation before: the certainty that an $\mathrm{X}$ result will be delivered. Reputational risks are mainly perceived by researchers that intend their work as a sort of contractual obligation, while do not represent an issue at all for researchers that consider their work as a mission. Style of thinking and personality resources are called to overcome reputational risks, and to restore a more appropriate judgment based on obligation of means instead obligation of results.

\section{Routine-related risk}

\footnotetext{
${ }^{10}$ Non-European visiting researchers remarked that, in their country of origin, not delivering the specified outcome of public grants could cost them their job position. 3 interviews have been carried out in an Italian research centre, with no significant differences with the French case.
} 
Source: Another interesting source of risk emerged during the interview is related to the establishment of clear routines in performing the research. One of the modelers in our case study pointed out that there is some type of way to do research that can foster creativity, although those ways may present other drawbacks. He said that when he gets funds to establish a collaboration with other academics he usually learns more because he benefits from the experience of the other academician, while when he receive funds to work with the industry sector he acknowledges that the scientific level of the collaboration is very poor. Nevertheless, when he is involved in a project with the industry sector he is forced to change his routines in terms of what are the step to follow and he moves from the theory-based work typical of a modeler to a work devoted to applying the theory to find a practical solution to problems. In terms of knowledge, he learns less but he does something new, which intrinsically stimulate his motivation and his creative thinking, thus pointing out a possible trade-off between the expansion of the knowledge base and creativity.

Resources: Style of thinking and environment might play an important role in avoiding risks related to routinary tasks. An experimental work by Curci et al. (2017) reveals that people opened to new experience demonstrate a higher level of creativity. In this sense, environments that do not impose pre-ordered routing in the performance of a research can assure the degree of freedom that researchers need to experience in order to easily change their approach to research.

\section{The lesson learned from the research star}

During the interviews, the researcher who received the highest consideration in the nomination part of the survey was also one of the most prolific scientists of the whole lab. She declared that her projects were all creatives and that each research was part of a scientific path, that science means risk and that scientists have to take risks by definition. While, at the beginning, such assertion could sound surprising, in the answers she provided later in the 
interview we found evidence that no actions or behaviors of her professional life were not guided by powerful resources that allow her to take risks. In the first place, when the excellence of your scientific path is the goal, your motivation is equal in all your projects. She demonstrated a high level of pragmatism by acknowledging that collaborating with someone that provides you just a service and is not personally attached to the research is always problematic, but one can easily deal with it by making them actively participate in the research and by eliminating hierarchies (she intrinsically motivate them). Her strong personality and style of thinking made her say that if she would ever realize that the organization for which she works would force her to change her scientific trajectory, she will change organization. She told us examples of how she is ready to cut relationship in a snap when she feels that her intellectual honesty is endangered (for instance when she is forced to add ghost-authors to papers). Moreover, she affirmed that she does not have any problem in letting Ph.D. students working on risky projects:

"we support thesis, not just publications... here we stimulate students to take some risks to show them what one can get in return".

\section{Conclusion}

In this paper we listed the risks individuated during interviews with scientists working in several fields of Hard Science, involved both in basic and applied research. Some of the highlighted risks affect to different extent researchers according to their status and their specific type of research activity. However, irrespective to field and nature, a great source of risks is represented by the need to organize the scientific research according to project management routines, which makes public research entities behaving like firms. Research proposals go through a sort of FFE phase (Koen, 2001), where risky ideas (hence potentially more creative) are rejected. The application of project management approaches to research seems to represent a barrier to creativity and breakthrough innovations. If research requires a market in which the 
future ideas can have an application (market pulled innovation), the innovative process might slow down and become more and more incremental and less radical.

In a context of increasingly complexity of knowledge, resources scarcity and urgent needs of populations, technological innovation has been acknowledged as one of the key factors for sustainable development. However, many authors argued that there is a trade-off between the certainty of technological discoveries process and their rate of radicalism and ability of being pervasive. (Benner \& Tushman, 1993; Smith \& Tushman, 2005). The recent transformation of the global economy have extended the concept of project management to public research in order to force researchers to comply with efficiency requirements, clear commitments towards given accomplishments and resource and time constraints. The project management approach, which is commonly applied in the management of research occurring in the private sector, introduces a number of formal procedures meant to prevent (or decrease the risk of) research dead-ends. As a consequence, objective measures and criteria have been individuate to make research evaluation feasible. Even so, the success of this transformation highly depends on the capacity of work environment to encourage compliance with economic goals, without killing researchers' creativity and their intellectual self-determination. Being creative involve different degrees of uncertainty in time and effectiveness of deliverables that may cause a failure of a research project. At the same time, reducing the risk of failure may represents a barrier to someone's creativity.

Rational economic agents usually operate within specific goals and, in pursuing them, risk minimization assessments lead to discarding highly daring ideas. In this process, creative ideas which are usually characterized by high level of risk - are often discarded in favour of less innovative ones.

In the light of this, the present study help the development of a management framework by individuating the source of creativity-related risks that can affect public researchers in the field of Hard Science. 
In the case studies analysed in this paper we found that risks have a different impact on scientific creativity, when it is considered as an ensemble of three different components: originality, plausibility and validity. Many of the risks observed during the interview of 27 scientists, were introduced at institutional level with the aim of fostering plausibility, validity, applicability and certainty in delivery objects and time. Although not intended to undermine originality, such procedures have been identified as one of the main source of risks for researchers' creativity.

The risks reported in the case study varied greatly according to two main variables: scientific field (theoretical vs experimental) and nature of research (basic vs applied).

Scientific field have a massive impact in terms of financial requirement, hence risks related to this issue are greater for expensive researches (experimental). At the same time, nonmarket oriented research are less endangered in terms of originality, but they struggle to be financed.

Basic research in Hard Science is characterized by high uncertainty in terms of economic returns of investments, which are mostly public grants. Hence, general economic conditions play a crucial role in influencing the types of research that are made. In the last decade, basic research started facing severe financial constraints, and resources were redirected towards more applied research projects showing higher chance of tangible success and evident public interest. In this context, creative thinking is threatened by financial constraints and uniformity and the high specificity (in terms of length of the research project and expected results) of research grants.

The interviews clarified that there is a dramatic difference between facilitating creativity in basic and applied science, in particular because scientists seem to have two different goals in mind. While the formers have to deal with major budget constrains due to lack of non-marketoriented-research-funds, the latter suffer from several limitations introduced by projects specifications required by projects calls. 
A main evidence arisen in this work is that researchers in our sample show a sort of parental love ${ }^{11}$ towards their creative projects, which can be led back to intrinsic motivators. Selfdetermination in choosing the subject, raising funds and developing the idea, sense of responsibility towards the project itself, as well as personal attachment to it, are the strongest and more diffuse elements observed during the interviews.

The following table summarizes and classifies the risks emerged during the interviews, as well as indicated the source of the risk, the category most affected and the managerial implications.

\footnotetext{
${ }^{11}$ Emotional feelings of different nature were reported by several researchers: i) the attachment due to the fact that the idea is your own brain baby, ii) the pain when the project was struggling, iii) the perseverance when there were no results, and vi) the pride to be the parent of the new-born creative idea.
} 


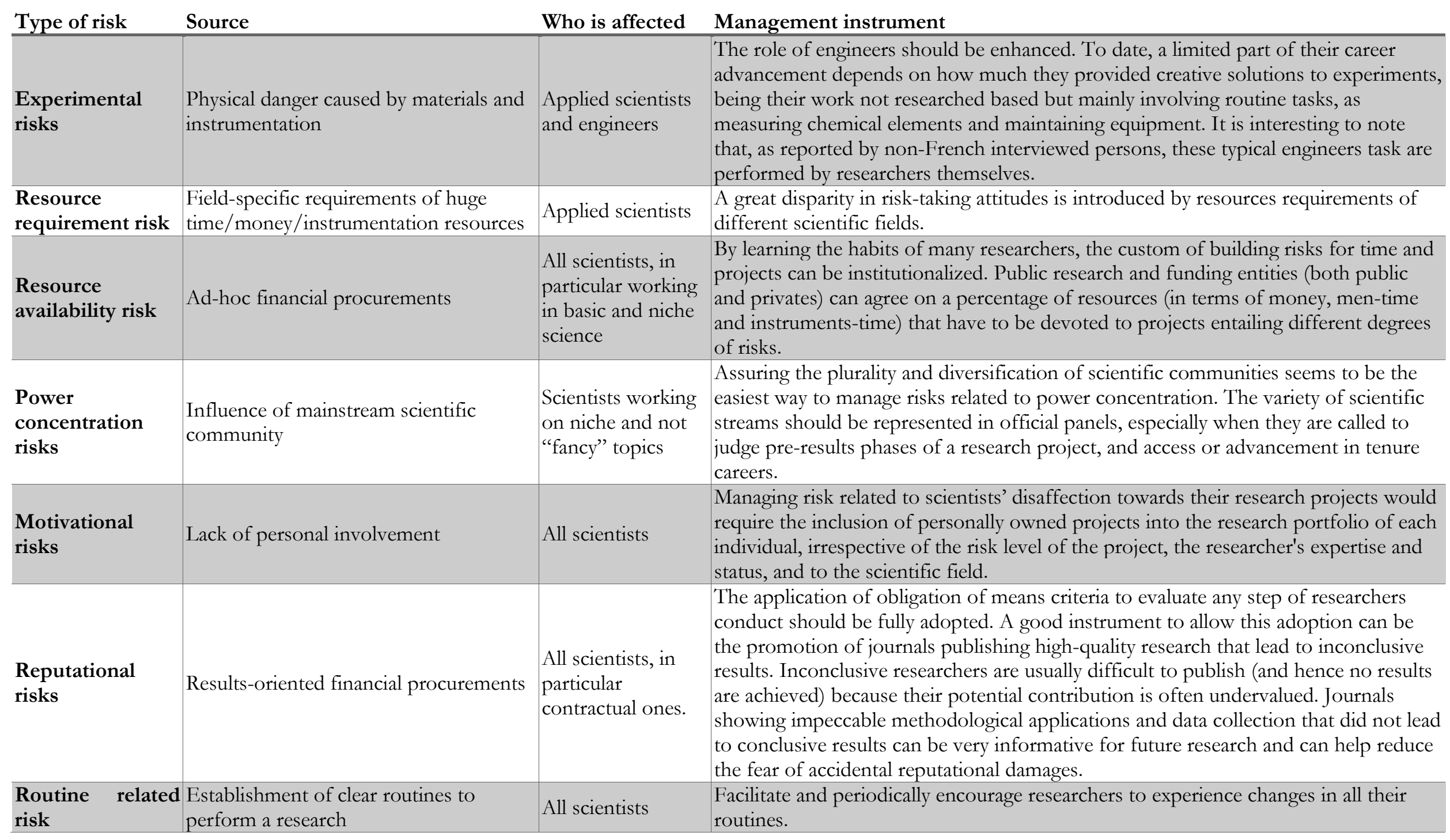




\section{References}

Amabile, T. M. (1983). The social psychology of creativity: A componential conceptualization. Journal of personality and social psychology, 45(2), 357.

Amabile, T. M. (1985). Motivation and creativity: Effects of motivational orientation on creative writers. Journal of personality and social psychology, 48(2), 393.

Amabile, T. M., Conti, R., Coon, H., Lazenby, J., \& Herron, M. (1996). Assessing the work environment for creativity. Academy of management journal, 39(5), 1154-1184.

Amabile, T. (2012). Componential theory of creativity. Harvard Business School.

Barczak, G., Lassk, F., \& Mulki, J. (2010). Antecedents of team creativity: An examination of team emotional intelligence, team trust and collaborative culture. Creativity and Innovation Management, 19(4), 332-345.

Barron, F., \& Harrington, D. M. (1981). Creativity, intelligence, and personality. Annual review of psychology, 32(1), 439-476.

Benner, M. J., \& Tushman, M. L. (2003). Exploitation, exploration, and process management: The productivity dilemma revisited. Academy of management review, 28(2), 238-256.

Caniëls, M. C., De Stobbeleir, K., \& De Clippeleer, I. (2014). The antecedents of creativity revisited: A process perspective. Creativity and Innovation Management, 23(2), 96-110.

Carver, C. S., \& White, T. L. (1994). Behavioral inhibition, behavioral activation, and affective responses to impending reward and punishment: the BIS/BAS scales. Journal of personality and social psychology, 67(2), 319.

Charness, G., \& Grieco, D. (2013). Individual Creativity, Ex-ante Goals and Financial Incentives. Department of Economics, UC Santa Barbara.

Cohendet, P., Harvey, J. F., \& Simon, L. (2013). Managing creativity in the firm. In BurgerHelmchen, T. The Economics of Creativity: Ideas, Firms and Markets, pp. 131-150.

Deci, E. L. (1971). Effects of externally mediated rewards on intrinsic motivation. Journal of personality and Social Psychology, 18(1), 105.

Deci, E. L., Koestner, R., \& Ryan, R. M. (2001). Extrinsic rewards and intrinsic motivation in education: Reconsidered once again. Review of educational research, 71(1), 1-27.

Dewett, T. (2004). Employee creativity and the role of risk. European Journal of Innovation Management, 7(4), 257-266.

Frey, S. B. (1997). Not Just for the Money. Edward Elgar Publishing Ltd., Cheltenham.

Frey, B. S., \& Jegen, R. (2001). Motivation Crowding Theory. Journal of Economic Surveys, 15(5), 589-611.

Gido, J., \& Clements, J. (2014). Successful project management. Cengage Learning.

Glover, J. A. (1977). Risky shift and creativity. Social Behavior and Personality: an international journal, $5(2), 317-320$.

Golann, S. E. (1962). The creativity motive. Joumal of personality, 30(4), 588-600.

Guilford, J. P. (1950). Creativity. American Psychologist, 5, 444-454. 
Hollingsworth, R. (2004). Institutionalizing excellence in biomedical research: the case of Rockefeller University, in D.H. Stapleton (Ed.), Creating a Tradition of Biomedical Research. Contributions to the History of the Rockefeller University, Rockefeller University Press, New York (2004), pp. 17-63.

Heinze, T., Bauer, G. (2007a). Characterizing creative scientists in nano S\&T: productivity, multidisciplinarity, and network brokerage in longitudinal perspective. Scientometrics, 70 (3), 811-830.

Heinze, T., Shapira, P., Senker, J. M., Kuhlmann, S. (2007b). Identifying creative research accomplishments: methodology and results for nanotechnology and human genetics. Scientometrics, 70 (1), pp. 125-152.

Heinze, T., Shapira, P., Rogers, J. D., Senker, J. M. (2009). Organizational and institutional influences on creativity in scientific research. Research Policy, 38(4), 610-623.

Jensen, H. S. (2011). The role of ambiguity in innovation. In 2nd conference on rhetoric and narratives in management research: Management and persuasion (pp. 49-58).

Keltner, D., Gruenfeld, D. H., \& Anderson, C. (2003). Power, approach, and inhibition. Psychological review, 110(2), 265.

Knight, F. H. (2012). Risk, uncertainty and profit. Courier Corporation.

Koen, P., Ajamian, G., Burkart, R., Clamen, A., Davidson, J., D'Amore, R., \& Wagner, K. (2001). Providing Clarity and a Common Language to the. Research-Technology Management, 44(2), 4655.

Kreps, D. M. (1997). Intrinsic motivation and extrinsic incentives. The American Economic Review, 359-364

Lazaric, N., \& Raybaut, A. (2014). Do incentive systems spur work motivation of inventors in high tech firms? A group-based perspective. Journal of Evolutionary Economics, 24(1), 135-157.

Lee, F., Edmondson, A. C., Thomke, S., \& Worline, M. (2004). The mixed effects of inconsistency on experimentation in organizations. Organization Science, 15(3), 310-326.

Lubart, T. I., \& Sternberg, R. J. (1995). An investment approach to creativity: Theory and data. The creative cognition approach, 269-302.

Mainemelis, C. (2010). Stealing fire: Creative deviance in the evolution of new ideas. Academy of Management Review, 35(4), 558-578.

Mednick, S. A. (1962). The associative basis of the creative process. Psychological Review, 69, 220232.

Ochse, R. (1990). Before the gates of excellence: The determinants of creative genius. CUP Archive.

Owan, H., \& Nagaoka, S. (2011). Intrinsic and extrinsic motivation for inventors. RIETI Discussion Paper Series 11-E-022. March, 2011

Torrance, E. P. (1974). The Torrance Tests of Creative Thinking. Norms-Technical Manual. Research Edition. Verbal Tests, Forms A and B-Figural Tests, Forms $A$ and B. Princeton, NJ: Personnel Press.

Rosenberg, N. (1982). Inside the black box: technology and economics. Cambridge University Press.

Rosenberg, N. (1994). Exploring the black box: Technology, economics, and history. Cambridge University Press.

Schumpeter, J. A. (1939). Business cycles. New York: McGraw-Hill. 
Shalley, C. E., Zhou, J., \& Oldham, G. R. (2004). The effects of personal and contextual characteristics on creativity: Where should we go from here?. Journal of management, 30(6), 933-958.

Simonton, D. K. (1988). Scientific genius. Cambridge University Press.

Simonton, D.K. (2004). Creativity in Science: Chance, Logic, Genius, and Zeitgeist. Cambridge University Press, Cambridge, UK (2004).

Smith, W. K., \& Tushman, M. L. (2005). Managing strategic contradictions: A top management model for managing innovation streams. Organization science, 16(5), 522-536.

Staw, B. M. (1989). Intrinsic and extrinsic motivation. Readings in managerial psychology, 197-209.

Staw, B. M. (1995). Why no one really wants creativity. Creative action in organizations, 161-66.

Sternberg, R. J. \& Lubart, T. I. (1991). An investment theory of creativity and its development. Human Development, 34, 1-31.

Sternberg, R. J., O'Hara, L. A., \& Lubart, T. I. (1997). Creativity as investment. California Management Review, 40, 8-21.

Torrance, E. P. (1988). The nature of creativity as manifest in its testing. The nature of creativity, 4375.

Woodman, R. W., Sawyer, J. E., \& Griffin, R. W. (1993). Toward a theory of organizational creativity. Academy of management review, 18(2), 293-321.

Zhong, C. B., Dijksterhuis, A., \& Galinsky, A. D. (2008). The merits of unconscious thought in creativity. Psychological Science, 19(9), 912-918. 